\title{
Detection of Chronic Kidney Disease by Using Different Equations of Glomerular Filtration Rate in Patients with Type 2 Diabetes Mellitus: A Cross-Sectional Analysis
}

\author{
Sojib Bin Zaman ${ }^{1}$ \\ 1. Faculty of Public Health, Khon Kaen University, Thailand \\ $\square$ Corresponding author: Sojib Bin Zaman, sojibbz@gmail.com \\ Disclosures can be found in Additional Information at the end of the article
}

\section{Abstract}

Introduction

Chronic kidney disease (CKD) is a global threat due to its high mortality. It is essential to know the actual magnitude of diabetic CKD to design a specific management program. However, there is limited knowledge regarding the most suitable equation to measure CKD in patients with Type 2 diabetes mellitus (T2DM). This paper aimed to analyze estimated glomerular filtration rate (eGFR) based on different equations to detect the CKD among T2DM.

Methods

A hospital-based cross-sectional study was carried out, and a clinical registry was used to collect 4,042 T2DM patients from a large district hospital in Northeast Thailand. CKD patients were diagnosed when eGFR was less than $60 \mathrm{ml} / \mathrm{min} / 1.73 \mathrm{~m}^{2}$. Using Stata statistical software (StataCorp LP, College Station, TX), three standard equations, such as 'modification of diet in renal disease (MDRD-4)', 'chronic kidney disease epidemiology collaboration (CKD-EPI)', and 'Cockcroft-Gault (C-G)' equations, were used to produce eGFR values to report and compare stages of CKD.

Results

The mean age of the patients was $61.4( \pm 10.7)$ years and male to female ratio was 1:1.9. According to the MDRD-4, CKD-EPI, and C-G equation, the prevalence of diabetic CKD was $21.4 \%, 21.9 \%$, and $31.4 \%$, respectively, and the frequency of CKD Stage 3 to 5 was found to be different among T2DM. About 3,789 (93.9\%) measurements appeared to be classified as different stages of CKD (Stages 1 to 5) between MDRD-4 and CKD-EPI equations (kappa: 0.905; $95 \%$ confidence interval (CI): 0.83 - 0.97, p < 0.001). However, this study found that the abovementioned agreement was $70.9 \%$ between CKD-EPI and C-G equation (kappa: 0.56, 95\% CI: $0.44-0.67, \mathrm{p}<0.001)$.

Conclusions

CKD-EPI equations can overcome the constraint of MDRD-4 and C-G equations to report CKD and can be used in patients with T2DM.

\section{How to cite this article}

Zaman S (June 14, 2017) Detection of Chronic Kidney Disease by Using Different Equations of Glomerular Filtration Rate in Patients with Type 2 Diabetes Mellitus: A Cross-Sectional Analysis . Cureus 9(6): e1352. DOI 10.7759/cureus.1352 
Categories: Endocrinology/Diabetes/Metabolism, Nephrology, Public Health

Keywords: diabetes mellitus, egfr, thailand, ckd

\section{Introduction}

The prevalence of chronic kidney disease (CKD) has been estimated to be more than 13\% globally [1-3]. According to statistics, approximately 200 million people suffer from CKD around the world today [4]. Diabetes mellitus is one of the leading causes of CKD around the world and responsible for causing high morbidity [5]. CKD might progress to renal failure, which can cause life-threatening complications for diabetic patients [6]. Dialysis, hemofiltration, and kidney transplantation are the available treatment options for the patients with CKD. However, this treatment protocol cannot reverse healthy kidney function back to normal but rather delays the progression of kidney damage [7]. CKD, as a complication of Type 2 diabetes mellitus (T2DM), has frequently been reported in Southeast Asian countries like Thailand [8]. Thailand is also geographically connected to the 'stone belt' zone where the renal stones are more common; this zone extends from the Central Asia to South Asia [9-10]. Therefore, kidney diseases due to T2DM or non-diabetic causes are common among the Thai population. However, the high burden of CKD due to T2DM poses a significant challenge to the Thai universal health coverage (UHC) system [11], which reiterates the importance of being aware of the recent diabetic CKD prevalence in Thailand.

Traditionally, serum creatinine concentration is used to diagnose CKD in many resource-poor settings [12]. However, the detection of CKD, depending on serum creatinine levels, might not be accurate when compared with other diagnostic approaches [13]. To illustrate, for older people and lean young females, this test is not a useful tool to detect renal disease [14]. Patients with reduced muscle mass may be related to reduced serum creatinine levels in their body [14-15]. Therefore, elderly patients might show lower serum creatinine concentrations despite having advanced kidney disease [13]. Direct measurement of the glomerular filtration rate (GFR) is reliable, but the procedure is cumbersome and expensive and hence, not very suitable in clinical settings. The alternate option is to use an estimated glomerular filtration rate (eGFR) rather than direct measurement [16]. Studies have used "modification of diet in renal disease - four variables (MDRD-4)", or "chronic kidney disease - epidemiology collaboration (CKD - EPI)", or "Cockcroft-Gault (C-G)" equations to report CKD [17]. Therefore, the reported CKD prevalence usually varies across the countries [18]. Some countries use "endstage renal disease (ESRD)" to report the prevalence of CKD as well [19]. The exact estimation of the number of ESRD patients due to T2DM is crucial to know, as the ESRD patients are likely to utilize dialysis services or kidney transplantation in near future [19]. However, there are controversies in deciding which method is more accurate to report the prevalence of CKD among the diabetic patients. The aim of this study was to analyze eGFR in patients with T2DM and to evaluate different equations to detect CKD in patients with T2DM.

\section{Materials And Methods}

\section{Study design and data source}

The Institutional Review Board of Khon Kaen University (KKU) approved this study (approval \#HE2247). This study performed a hospital-based cross-sectional study. Data was obtained from the clinical registry of diabetic patients who received medical care from a large district hospital in Northeast of Thailand. Based on the eligibility criteria, data was obtained from 4,042 T2DM patients between January 01, 2015 and December 31, 2015. A medical record number was used as a unique identifier to connect hospital records across the hospital's electronic health record system. Pre-diagnosed diabetic patients who obtained a serum creatinine test in the hospital during the study period and were 18 years of age or older were included in the study. Patients 
with Type 1 or gestational diabetes mellitus were excluded from this study.

\section{Definition of CKD and its staging}

This study used both conventional creatinine measurement and eGFR equations to determine CKD. A traditional cut-off point of serum creatinine $(\geqslant 1.4 \mathrm{mg} / \mathrm{dL}$ if male and $\geqslant 1.2 \mathrm{mg} / \mathrm{dL}$ if female) was considered to detect CKD. In this study, CKD was defined based on the eGFR value below $60 \mathrm{~mL} / \mathrm{min} / 1.73 \mathrm{~m} 2$. The criteria were set according to the "National Kidney Foundation Kidney Disease Outcomes Quality Initiative (NKF-KDOQI)” [17]. The staging of CKD was categorized according to NKF-KDOQI criteria based on different eGFR distribution: Stage 1: > $90 \mathrm{~mL} / \mathrm{min}$; Stage 2: 60 - $89 \mathrm{~mL} / \mathrm{min}$; Stage 3: $30-60 \mathrm{~mL} / \mathrm{min}$; Stage 4: 15-29 mL/min; and Stage $5:<15 \mathrm{~mL} / \mathrm{min}$.

\section{Estimated eGFR equations}

This study has used "modification of diet in renal disease - four variable (MDRD-4)", "chronic kidney disease - epidemiology collaboration (CKD - EPI)", and "Cockcroft-Gault (C-G)" equations to calculate eGFR to report CKD.

i. Four-variable MDRD equation [7]

eGFR $=186.3$ x S. Cr (mg/dL) - 1.154 x age (year) - 0.203 ( x 0.742 for women) (x 1.21 for nonHispanic Black)

Here, S. Cr is serum creatinine in $\mathrm{mg} / \mathrm{dL}$

\section{ii. CKD-EPI equation [17]}

For females with S. Cr $\leqslant 62 \mu \mathrm{mol} / \mathrm{L}: \mathrm{eGFR}=(144+22$ if Black $) \mathrm{x}(\mathrm{Cr} / 0.7)^{\wedge}-0.329 \times 0.993^{\wedge}$ age

For females with S. Cr > $62 \mu \mathrm{mol} / \mathrm{L}:$ eGFR $=(144+22$ if Black $) \times(\mathrm{Cr} / 0.7)^{\wedge}-1.209 \times 0.993^{\wedge}$ age

For males with S. $\mathrm{Cr} \leqslant 80 \mu \mathrm{mol} / \mathrm{L}: \quad$ eGFR $=(141+22$ if Black $) \times(\mathrm{Cr} / 0.9)^{\wedge}-0.411 \times 0.993^{\wedge}$ age

For males with S. Cr > $80 \mu \mathrm{mol} / \mathrm{L}: \quad$ eGFR $=(141+22$ if Black $) \times(\mathrm{Cr} / 0.9)^{\wedge}-1.209 \times 0.993^{\wedge}$ age

Here, the unit of $\mathrm{S}$. Cr is in $\mu \mathrm{mol} / \mathrm{L}$

iii. Cockcroft-Gault equation [20]

eGFR $=(140$ - age $) \times$ weight $x 1.04$ (if female $) /$ S. Cr, and

eGFR $=(140-$ age $) \times$ weight $x 1.23($ if male $) / S . C r$

Here, the unit of S. Cr is in $\mu \mathrm{mol} / \mathrm{L}$ and unit of weight is by kilograms.

\section{Data management and statistical analysis}

Data entry was performed by a group of skilled data operator under the supervision of a data management officer. Errors in data entry were revised after cross-checking both the laboratory records and clinical case recording forms. Frequencies and proportions were used to present categorical variables. Mean and standard deviation (SD) were considered to describe continuous 


\section{Cureus}

variables. eGFR was estimated by using serum creatinine and additional covariates (age, sex, body weight, and non-Hispanic Black). Three standard equations (CKD-EPI, MDRD-4, and C-G) were used to produce eGFR values. Mann-Whitney $U$ test was used to find out the comparison of different eGFR values. The kappa index was used to analyze the level of agreement to determine CKD stages, which were obtained by using three equations. However, in the absence of a gold standard method to estimate GFR, this study compared eGFR values obtained among the CKD-EPI, MDRD-4, and C-G equations. Stata, version 13 special edition (College Station, Texas, USA), was used for analyzing the data considering $\mathrm{p}$-value $<0.05$.

\section{Results}

\section{Basic information about patient characteristics}

The mean age of the T2DM patients was $61.4( \pm 10.7)$ years, and the male to female ratio was 1:1.9. Respondents' occupation were farmers (59.4\%), different types of employment (28\%), including day laborer, housewife, government employee, soldier, monk, etc., and 10.7\% were unemployed. Only $1.3 \%$ participants were identified as current alcohol drinkers, and $3.8 \%$ of T2DM patients gave a positive history of current smoking. The mean serum triglycerides and low-density lipoprotein cholesterol (LDL-C) was $179 \mathrm{mg} / \mathrm{dl}$ and $110 \mathrm{mg} / \mathrm{dl}$, respectively. Every diabetic patient was treated under the three schemes (the universal coverage scheme, Civil Service welfare, and Social Security scheme) of the Universal Health Coverage (UHC) who attended the participating hospital (Table 1).

\section{Characteristics}

Age

Mean \pm SD

Min : Max

Sex

Male

Female

2,663

Universal Health Coverage (UHC)

Universal coverage scheme

3,333

Civil Service welfare

486

Social Security scheme

223

Occupation

Farmer

Different categories

1,110

Unemployment

434

Body Mass Index

Mean \pm SD
34.2

65.8

82.5

5.5

10.7
$24.9 \pm 4.0$

\section{Number}

\section{Percentage}




\section{Cureus}

Min : Max

Characteristics

Hypertension

No

Yes

Serum Triglyceride

Mean \pm SD

Min : Max

Glycated Hemoglobin (HbA1c)

Mean \pm SD

Min: Max

LDL - Cholesterol

Mean \pm SD

Min : Max

Current Smoker

No

Yes

Current Alcohol Drinker

No

Yes
$14.7-40.7$

Number

Percentage

1,729

42.8

2,313

57.2

$179.18 \pm 108.42$

$24-947$

$8.46 \pm 2.19$

$4.4-21$

$110.14 \pm 33.19$

$78-578$

3,885

96.2

157

3.8

3,986

98.6

56

1.4

TABLE 1: Basic Information About the Patient Characteristics $(n=4,042)$

Categorical data are presented as number (percentage); continuous data are shown as means \pm SD.

SD: standard deviation; LDL: low-density lipoprotein

\section{Prevalence of T2DM patients based on conventional method and eGFR equations}

About 18.6\% patients were categorized as CKD based on serum creatinine concentration. CKD was slightly higher among the males (19.4\%) as compared to the females (18.2\%) (Table 2). 


\section{Cureus}

\begin{tabular}{|c|c|c|}
\hline Serum Creatinine (mg/dl) & Number & Percentage \\
\hline Male $\geq 1.4 \quad(n=1,379)$ & 268 & 19.4 \\
\hline Female $\geq 1.2 \quad(n=2,663)$ & 485 & 18.2 \\
\hline$\geq 1.4$ if Male and $\geq 1.2$ if Female & 753 & 18.6 \\
\hline
\end{tabular}

\section{TABLE 2: CKD According to Elevated Serum Creatinine Level}

CKD: chronic kidney disease

According to the MDRD-4, CKD-EPI, and C-G equation, the mean value of eGFR was 83.9 $\mathrm{mL} / \mathrm{min} / 1.73 \mathrm{~m} 2,78.7 \mathrm{~mL} / \mathrm{min} / 1.73 \mathrm{~m} 2$, and $75.3 \mathrm{~mL} / \mathrm{min} / 1.73 \mathrm{~m} 2$, respectively, among the T2DM patients $(\mathrm{p}<0.05)$. About $21.9 \%$ patients were classified as CKD based on the CKD-EPI equation. The frequency of CKD Stages 3 to 5 was found to be dissimilar, considering the different equations. After the application of the MDRD-4 equation, the proportion of CKD was 21.4\% (Table 3). The CKD-EPI equation driven CKD number was slightly higher in Stages 4 and 5 when compared with MDRD-4. However, the frequency of CKD was 1,272 (31.4\%) according to the $C-G$ equation. Patients with Stage $3(n=1,040)$ were much higher based on the $C-G$ equation as compared to the MDRD-4 $(n=727)$ and the CKD-EPI equation $(n=730)$ (Figure 1$)$.

CG Formula

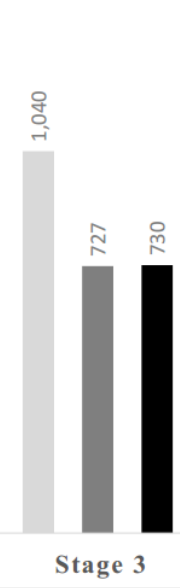

MDRD 4
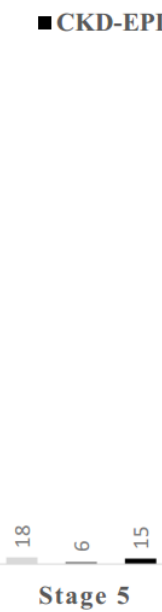

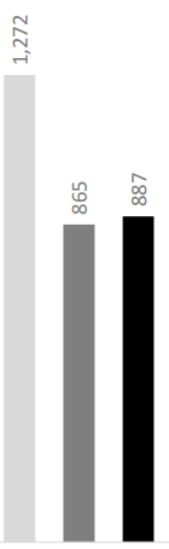

Stage 3 to 5

FIGURE 1: CKD distribution according to C-G, MDRD-4, and CKD-EPI equations

CG: Cockcroft- Gault equation; CKD: chronic kidney disease; CKD-EPI: chronic kidney disease epidemiology; eGFR: estimated glomerular filtration rate; MDRD-4: modification of diet in renal disease (4 inputs, simplified) 


\section{Cureus}

However, the frequency of Stage 5 CKD patients was $0.37 \%$ and $0.15 \%$ considering the CKD-EPI and MDRD-4 equation, respectively, and it was found to be statistically significant (Table 3).

\begin{tabular}{|c|c|c|c|c|}
\hline CKD Stage & eGFR $\left(\mathrm{mL} / \mathrm{min} / 1.73 \mathrm{~m}^{2}\right)$ & C-G Number (\%) & MDRD-4 Number (\%) & CKD-EPI Number (\%) \\
\hline 1 & $>90$ & $1,634(40.4)$ & $1,997(49.4)$ & $2,021(50.0)$ \\
\hline 2 & $60-89$ & $1,136(28.1)$ & $1,180(29.2)$ & $1,134(28.1)$ \\
\hline 3 & $30-60$ & $1,040(25.7)$ & $727(17.9)$ & $730(18.1)$ \\
\hline 4 & $15-29$ & $214(5.2)$ & 132 (3.2) & 142 (3.5) \\
\hline 5 & $<15$ & $18(0.4)$ & $6(0.1)$ & $15(0.3)$ \\
\hline CKD (3-5) & $<60 \mathrm{~mL} / \mathrm{min} / 1.73 \mathrm{~m}^{2}$ & $1,272(31.4)$ & $865(21.4)$ & $887(21.9)$ \\
\hline
\end{tabular}

\section{TABLE 3: Prevalence of CKD Based on Different eGFR Equation}

CKD: chronic kidney disease; eGFR: estimated glomerular filtration rate; MDRD-4: modification of diet in renal disease (4 inputs, simplified); CKD-EPI: chronic kidney disease epidemiology; CG: Cockcroft- Gault equation

\section{Comparisons of different eGFR equations}

Of the 4,042 measurements, 3,789 (93.9\%) values were similarly classified as Stages 1 to 5 with MDRD-4 and CKD-EPI equations. The reported kappa index between the two equations, MDRD-4 and CKD-EPI, was 0.90 (95\% confidence interval (CI): $0.83-0.97, \mathrm{p}<0.001$ ) (Table 4). However, there was a 6.1\% disagreement in classifying the CKD stages between two equations. About 28 patients were classified as Stages 4 and 5 using the CKD-EPI equation, but these patients actually were not the above-mentioned stages when we considered the MDRD-4 equation to detect the CKD stage. 


\section{Cureus}

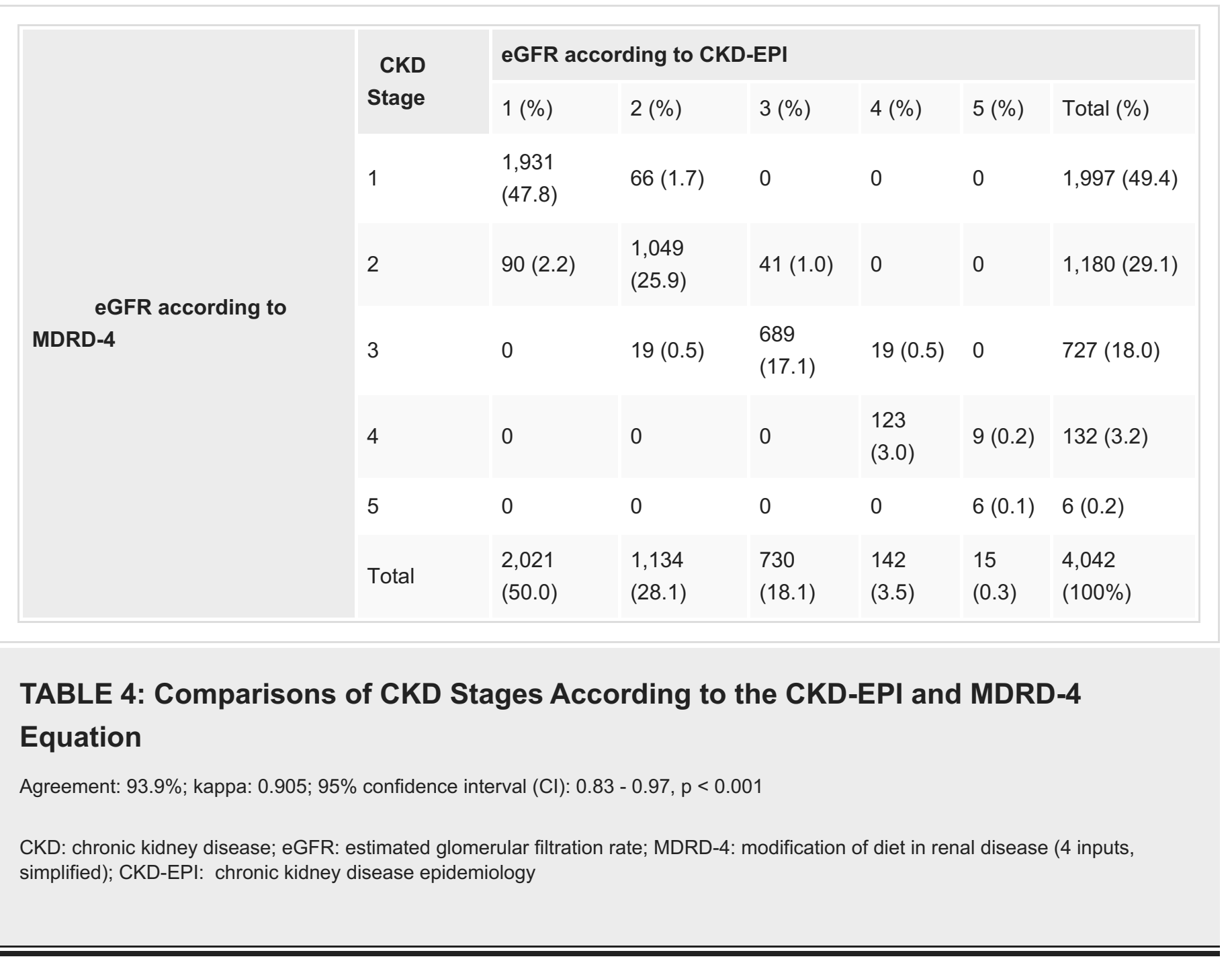

However, this study found that agreement between the CKD-EPI and C-G equation was 2,869 (70.9\%) with a kappa index of 0.56 (95\% CI: $0.44-0.67, \mathrm{p}<0.001)$ (Table 5). Also, the agreement was found to be only $69.6 \%$ (kappa: $0.54,95 \%$ CI: $0.44-0.67, \mathrm{p}<0.001$ ) after comparing the MDRD-4 and C-G equation (data not shown). 


\section{Cureus}

\begin{tabular}{|c|c|c|c|c|c|c|c|}
\hline \multirow{8}{*}{$\begin{array}{l}\text { eGFR according to CKD- } \\
\text { EPI }\end{array}$} & \multirow{2}{*}{$\begin{array}{l}\text { CKD } \\
\text { Stage }\end{array}$} & \multicolumn{6}{|c|}{ eGFR according to C-G } \\
\hline & & $1(\%)$ & $2(\%)$ & $3(\%)$ & $4(\%)$ & $5(\%)$ & Total (\%) \\
\hline & 1 & $\begin{array}{l}1,534 \\
(37.9)\end{array}$ & $474(11.7)$ & $13(0.3)$ & 0 & 0 & $2,021(50.0)$ \\
\hline & 2 & $80(1.9)$ & $623(15.4)$ & $431(10.6)$ & 0 & 0 & 1,134 (28.1) \\
\hline & 3 & $17(0.4)$ & $39(0.9)$ & $584(14.4)$ & $90(2.2)$ & 0 & $730(18.1)$ \\
\hline & 4 & 0 & 0 & $14(0.3)$ & $\begin{array}{l}119 \\
(2.9)\end{array}$ & $9(0.2)$ & $142(3.6)$ \\
\hline & 5 & 0 & 0 & 0 & $6(0.1)$ & $9(0.2)$ & $15(0.4)$ \\
\hline & Total & $\begin{array}{l}1,631 \\
(40.4)\end{array}$ & $\begin{array}{l}1,136 \\
(28.1)\end{array}$ & $\begin{array}{l}1,042 \\
(25.8)\end{array}$ & $\begin{array}{l}215 \\
(5.3)\end{array}$ & $\begin{array}{l}18 \\
(0.4)\end{array}$ & $4,042(100 \%)$ \\
\hline
\end{tabular}

\section{TABLE 5: Comparisons of CKD Stages According to CKD-EPI and C-G Equation}

Agreement: 70.9\%; kappa: 0.56; 95\% confidence interval $(\mathrm{Cl}): 0.44-0.67, \mathrm{p}<0.001$

CKD: chronic kidney disease; eGFR: estimated glomerular filtration rate; MDRD-4: modification of diet in renal disease (4 inputs, simplified); CKD-EPI: chronic kidney disease epidemiology; C-G: Cockcroft-Gault equation

\section{Discussion}

This study found that $21.9 \%$ of T2DM patients were detected as CKD according to the CKD-EPI equation. However, the frequency of CKD was $31.4 \%$ and $21.4 \%$ based on the $\mathrm{C}-\mathrm{G}$ and MDRD-4 equation, respectively. Therefore, MDRD-4 and CKD-EPI methods were coherent to report the CKD prevalence with a kappa index of 0.90 . Based on the concentration of serum creatinine, the CKD was found to be $18.6 \%$ among the T2DM patients. Therefore, underestimation or overestimation of the prevalence of CKD due to the application of different detection methods has emerged as a future challenge for many developing countries [21]. However, the frequency of CKD in this study was found to be smaller in comparison to previous figures, which was $27 \%$ in 2008 [22].

Considering the $\mathrm{C}-\mathrm{G}$ equation, the number of CKD patients was overestimated (31\%); this was found almost similar with other research findings [22]. One of the main disadvantages of the C$\mathrm{G}$ equation is the requirement of patient's body weight, which may not always be available in a laboratory set up [23]. On the other hand, the MDRD-4 equation is most familiar among the nephrologists and globally known as a cornerstone method to report CKD [17]. However, the MDRD-4 equation is not without drawbacks as it can offer a false positive approximate when patients present with high eGFR values (Stages 4-5). This study found a reliable percentage of CKD (Stages 3 to 5) after using the CKD-EPI equation, and these findings are consistent with a previous study [24]. Therefore, the CKD-EPI equation might be considered as a better tool to detect the Stage 5 CKD patient when compared with MDRD-4, which supports similar arguments [25]. The CKD-EPI equation is found to be more consistent than the MDRD-4 equation, and it has been found that application of CKD-EPI has increased recently for the clinical settings. However, CKD-EPI still needs validation before starting the use of the routine clinical tests [17]. 
In our study, according to CKD-EPI equation, the frequency of CKD patients was slightly larger in Stage $5(0.37 \%)$ compared to the MDRD-4 $(0.15 \%)$. However, there are still controversies to determine which method is better to estimate GFR among the Asians [26]. Our findings are consistent with the previous study where Satirapoj, et al. compared the performance of different methods to identify kidney disease and found bias among the eGFR equations when comparing with direct GFR [27]. Diabetic patients are more vulnerable to develop CKD when compared with non-diabetic patients. A systematic review has shown that the mortality rate of the patients with Stage 3 CKD is higher than those without CKD [28]. Another review has shown that half of the patients suffering from Stage 3 CKD have progressed to Stage 4 and Stage 5 over 10 years [29]. Eventually, these patients will need renal replacement therapy and kidney transplantation in near future. However, the progression of end-stage of CKD can be prolonged by proper detection of eGFR [30].

To the best of researcher's knowledge, there is no study so far conducted among T2DM patients in Thailand to evaluate the prevalence of kidney disease based on different eGFR equations. This study has found that the CKD-EPI equation can estimate kidney function better than the MDRD-4 and C-G equations, which also corresponds with previous research [27]. Therefore, use of the CKD-EPI equation or a country-specific validated eGFR equation will give the true prevalence of diabetic CKD patients. It will certainly help the policymakers to implement the inclusion of dialysis support services for the true positive CKD patients in Thailand.

\section{Limitation of the study}

Due to the absence of a gold standard GFR, it was hard to comment which method was more accurate when compared with different equations. Moreover, this study did not compare the performance of the various eGFR equations based on bias, precision, and accuracy.

\section{Conclusions}

eGFR equations can be a suitable method to estimate CKD as a comparison to the conventional serum creatinine measurement. This study found that CKD-EPI equation attempts to overcome the constraint of MDRD- 4 and $C-G$ equations to report CKD and might be suitable to use in patients with T2DM. However, a robust validation of CKD-EPI equation is warranted in Thailand.

\section{Additional Information Disclosures}

Human subjects: Consent was obtained by all participants in this study. Khon Kaen University Ethical Committee issued approval approval \#HE2247. The Ethical Committee of Khon Kaen University (KKU) had approved this study. The Director of the district hospital provided permission to use the hospital data. This study extracted unidentified data from routine electronic health records. The researcher did not contact any patient personally for data collection. Hence, the institutional review board of KKU waived the researcher from obtaining consents from individual patients. . Animal subjects: All authors have confirmed that this study did not involve animal subjects or tissue. Conflicts of interest: In compliance with the ICMJE uniform disclosure form, all authors declare the following: Payment/services info: All authors have declared that no financial support was received from any organization for the submitted work. Financial relationships: All authors have declared that they have no financial relationships at present or within the previous three years with any organizations that might have an interest in the submitted work. Other relationships: All authors have declared that there are no other relationships or activities that could appear to have influenced the submitted work. 


\section{Acknowledgements}

This research study was partially supported by the German Academic Exchange Service (DAAD). The author is grateful to Prof. Frank Peter Schelp, Prof. Pattara Sanchaisuriya, Wilaiphorn Thinkhamrop, Ina Hammesfahr, Matthias Borchert, Sheikh Mohammed Shariful Islam, Rajat Das Gupta, and Kazi Rezaul Ahsan for their advice and support. The author also would like to take this opportunity to thank all the staff from Khon Kaen University and Charite University Medicine Berlin.

\section{References}

1. Snyder JJ, Foley RN, Collins AJ: Prevalence of CKD in the United States: a sensitivity analysis using the National Health and Nutrition Examination Survey (NHANES) 1999-2004. Am J Kidney Dis. 2009, 53:218-28. 10.1053/j.ajkd.2008.07.034

2. Coresh J, Selvin E, Stevens LA, et al.: Prevalence of chronic kidney disease in the United States. JAMA. 2007, 298:2038-47. 10.1001/jama.298.17.2038

3. Hill NR, Fatoba ST, Oke JL, et al.: Global prevalence of chronic kidney disease-A systematic review and meta-analysis. PLoS One. 2016, 11:e0158765. 10.1371/journal.pone.0158765

4. Ojo A: Addressing the global burden of chronic kidney disease through clinical and translational research. Trans Am Clin Climatol Assoc. 2014, 125:229-46.

5. Ingsathit A, Thakkinstian A, Chaiprasert A, et al.: Prevalence and risk factors of chronic kidney disease in the Thai adult population: Thai SEEK study. Nephrol Dial Transplant. 2010, 25:1567-75. 10.1093/ndt/gfp669

6. Anand S, Khanam MA, Saquib J, et al.: High prevalence of chronic kidney disease in a community survey of urban Bangladeshis: a cross-sectional study. Global Health. 2014, 10:910. 10.1186/1744-8603-10-9

7. Wisplinghoff $\mathrm{H}$, Bischoff $\mathrm{T}$, Tallent SM, et al.: Nosocomial bloodstream infections in US hospitals: analysis of 24,179 cases from a prospective nationwide surveillance study. Clin Infect Dis. 2004, 39:309-17. 10.1086/421946

8. Muktabhant B, Sanchaisuriya P, Sarakarn P, et al.: Use of glucometer and fasting blood glucose as screening tools for diabetes mellitus type 2 and glycated haemoglobin as clinical reference in rural community primary care settings of a middle income country. BMC Public Health. 2012, 12:349. 10.1186/1471-2458-12-349

9. Jha V: End-stage renal care in developing countries: the India experience . Ren Fail. 2004, 26:201-208. 10.1081/JDI-120039516

10. Abbagani S, Gundimeda SD, Varre S, et al.: Kidney stone disease: Etiology and evaluation . Internatl J Applied Biol Pharmaceut Tech. 2010, 1:175-82.

11. Anutrakulchai S, Mairiang P, Pongskul C, et al.: Mortality and treatment costs of hospitalized chronic kidney disease patients between the three major health insurance schemes in Thailand. BMC Health Serv Res. 2016, 16:528. 10.1186/s12913-016-1792-9

12. Florkowski CM, Chew-Harris J: Methods of estimating GFR-different equations including CKD-EPI. Clin Biochem Rev. 2011, 32:75-79.

13. Swedko PJ, Clark HD, Paramsothy K, Akbari A: Serum creatinine is an inadequate screening test for renal failure in elderly patients. Arch Intern Med. 2003, 163:356-60. 10.1001/archinte.163.3.356

14. Lamb EJ, Webb MC, Simpson DE, et al.: Estimation of glomerular filtration rate in older patients with chronic renal insufficiency: is the modification of diet in renal disease formula an improvement?. J Am Geriatr Soc. 2003, 51:1012-17. 10.1046/j.1365-2389.2003.51330.x

15. Baxmann AC, Ahmed MS, Marques NC, et al.: Influence of muscle mass and physical activity on serum and urinary creatinine and serum cystatin C. Clin J Am Soc Nephrol. 2008, 3:348-54. 10.2215/CJN.02870707

16. Hsu CY, Bansal N: Measured GFR as "gold standard"--all that glitters is not gold? . Clin J Am Soc Nephrol. 2011, 6:1813-14. 10.2215/CJN.06040611

17. Levey AS, Stevens LA, Schmid CH, et al.: A new equation to estimate glomerular filtration rate. Ann Intern Med. 2009, 150:604-12. 10.7326/0003-4819-150-9-200905050-00006

18. Cristelli M, Cofán F, Rico N, et al.: Estimation of renal function by CKD-EPI versus MDRD in a cohort of HIV-infected patients: a cross-sectional analysis. BMC Nephrol. 2017, 18:58. 


\subsection{6/s12882-017-0470-4}

19. Domrongkitchaiporn S, Sritara P, Kitiyakara C, et al.: Risk factors for development of decreased kidney function in a southeast Asian population: a 12-year cohort study. J Am Soc Nephrol. 2005, 16:791-99. 10.1681/ASN.2004030208

20. Cockcroft DW, Gault MH: Prediction of creatinine clearance from serum creatinine . Nephron. 1976, 16:31-41. 10.1159/000180580

21. Zaman SB, Hossain N: Universal health coverage: A burning need for developing countries . J Med Res. 2017, 1:18-20. 10.5281/zenodo.546770

22. Narenpitak S, Narenpitak A: Prevalence of chronic kidney disease in type 2 diabetes in primary health care unit of Udon Thani province, Thailand. J Med Assoc Thai. 2008, 91:150513.

23. Akbari A, Swedko PJ, Clark HD, et al.: Detection of chronic kidney disease with laboratory reporting of estimated glomerular filtration rate and an educational program. Arch Intern Med. 2004, 164:1788-92. 10.1001/archinte.164.16.1788

24. Kaitwatcharachai C: Bedside renal assessment: a comparison of various prediction equations in Thai healthy adults. J Med Assoc Thai. 2006, 89:S146-50.

25. Sabanayagam C, Wong TY, Tai ES: The CKD-EPI equation and MDRD study equation find similar prevalence of chronic kidney disease in Asian populations. Ann Intern Med. 2009, 151:892-93. 10.7326/0003-4819-151-12-200912150-00014

26. Kitiyakara C, Yamwong S, Vathesatogkit P, et al.: The impact of different GFR estimating equations on the prevalence of CKD and risk groups in a Southeast Asian cohort using the new KDIGO guidelines. BMC Nephrol. 2012, 13:1. 10.1186/1471-2369-13-1

27. Satirapoj B, Jirawatsiwaporn K, Tangwonglert T, Choovichian P: Performance of the estimated glomerular filtration rate creatinine and cystatin $\mathrm{C}$ based equations in Thai patients with chronic glomerulonephritis. Int J Nephrol Renovasc Dis. 2015, 8:145-50. 10.2147/IJNRD.S93866

28. Sharma P, McCullough K, Scotland G, et al.: Does stage-3 chronic kidney disease matter? A systematic literature review. Br J Gen Pract. 2010, 60:e266-76. 10.3399/bjgp10X502173

29. Baek SD, Baek CH, Kim JS, et al.: Does stage III chronic kidney disease always progress to endstage renal disease? A ten-year follow-up study. Scand J Urol Nephrol. 2012, 46:232-38. 10.3109/00365599.2011.649045

30. Michels WM, Grootendorst DC, Verduijn M, et al.: Performance of the Cockcroft-Gault, MDRD, and new CKD-EPI formulas in relation to GFR, age, and body size. Clin J Am Soc Nephrol. 2010, 5:1003-1009. 10.2215/CJN.06870909 